\title{
ICRF Wave Field Measurements in the Presence of Scrape Off Layer Turbulence on the ASDEX Upgrade Tokamak a)
}

\author{
R. Ochoukov, ${ }^{1, b)}$ V. Bobkov, ${ }^{1}$ H. Faugel, ${ }^{1}$ H. Fünfgelder, ${ }^{1}$ J. Jacquot,${ }^{1}$ \\ J.-M. Noterdaeme, ${ }^{1,2}$ G. Suárez López, ${ }^{1,3}$ and ASDEX Upgrade Team ${ }^{1, c)}$
}

\author{
${ }^{1}$ Max Planck Institute for Plasma Physics, Boltzmannstr. 2, 85748 Garching, Germany \\ ${ }^{2}$ UGent, Applied Physics Department, 9000 Gent, Belgium \\ ${ }^{3}$ Ludwig Maximilian University of Munich, Professor-Huber-Platz 2, 80539 München, Germany \\ (Presented XXXXX; received XXXXX; accepted XXXXX; published online XXXXX) \\ (Dates appearing here are provided by the Editorial Office)
}

A new array of B-dot probes was installed on ASDEX Upgrade. The purpose of the new diagnostic is to study ICRF wave field distributions in the evanescent SOL plasma region on the low field side of ASDEX Upgrade. The vacuum measurements (no gas, $\mathrm{B}_{\mathrm{T}}=0 \mathrm{~T}$ ) reveal ICRF wave field measurements consistent with the profiles expected from the newly installed 3-strap ICRF antennas outside the antenna box: the shape of the toroidal distribution of both the amplitude and the phase is the same for the case of only the central straps being active, as for the case of only the side straps being active. These profiles become strongly modified during plasma operations. The modifications can be separated into two types: "Inter-ELM" and "During-ELM" periods. The phase distribution of the ICRF wave fields remains well-defined during the Inter-ELM period; however, it becomes more spread out over the entire $360^{\circ}$ range during ELMs. The observed modulations cannot be explained by the observed changes in the ICRF power, as monitored in the transmission line. However, they are consistent with ICRF coupling changes introduced by plasma filaments: the plasma density perturbations due to the filaments are high enough to change the nature of the fast ICRF wave field from evanescent to propagating. The coverage of the present diagnostic is being expanded to include both the low field side and the high field side probes. Additionally, a manipulator probe head is being developed to measure ICRF wave field radial profiles across the SOL region.

\section{INTRODUCTION}

Plasma heating using radio frequency (RF) waves in the Ion Cyclotron Range-off Frequencies (ICRF) band is a promising technique to reach fusion relevant temperatures in magnetized plasmas. However, the ICRF wave that is responsible for efficient core plasma heating, the fast wave ${ }^{1}$, is evanescent in low density plasma regions, the regions where ICRF wave launchers are located. As a result, the fast ICRF wave must tunnel from the launcher to the core plasma. This tunneling impacts fast wave coupling and, hence, net ICRF power transferred to the core in a magnetized plasma device, such as a tokamak.

A key feature of magnetically confined plasmas in tokamaks is the presence of turbulence ${ }^{2}$. Turbulence manifests itself in quasi-periodic fluctuations of key plasma properties: the plasma density $\mathrm{n}_{\mathrm{e}}$, the electron temperature $\mathrm{T}_{\mathrm{e}}$, the plasma potential and other parameters. While these fluctuations are present in both the confined (core) and unconfined (scrape-off layer or SOL) plasma regions, we are particularly interested in the unconfined SOL plasma region - the layer of plasma responsible for ICRF wave coupling. For the case of the SOL plasma region, the dominant turbulent structures are in the form of filaments or blobs ${ }^{3}$. These are field-aligned perturbations, which are responsible for

\footnotetext{
a) Invited paper published as part of the Proceedings of the 21st Topical Conference on High-Temperature Plasma Diagnostics (HTPD 2016) in Madison, Wisconsin, USA.

b) Author to whom correspondence should be addressed:

roman.ochoukov@ipp.mpg.de

${ }^{c}$ For authors list, see H. Zohm et al., Nucl. Fusion 55, 104010 (2015).
}

regulating heat and particle transport across the $\mathrm{SOL}^{3}$. RF wave dynamics in turbulent magnetized plasmas have been extensively examined both theoretically and experimentally ${ }^{4-8}$, with the RF waves in the lower hybrid frequency range receiving the most attention due to the wavelength size being comparable to the characteristic cross-field scale length of turbulent structures ${ }^{4,5,7,8}$. In our case, we are interested in studying the wave-turbulence interaction between ICRF waves and turbulence in the SOL, the region where the fast ICRF wave is typically evanescent ${ }^{9,10}$ and the plasma density perturbations introduced by turbulence are large enough that the fast wave becomes propagating within the filaments ${ }^{10}$.

The outline of the paper is as follows. First, we introduce a newly installed high-frequency B-dot (HFB) diagnostic ${ }^{11}$ dedicated to studying ICRF wave fields in the SOL region of ASDEX Upgrade. Next, we use the HFB diagnostic to characterize vacuum ICRF fields excited by the ASDEX Upgrade ICRF antennas in the torus and compare them to the fields expected from a COMSOL-based ${ }^{12}$ numerical model of the ASDEX Upgrade vacuum vessel. Finally, we look at the excited ICRF wave field structure during plasma operations, where both the amplitude, the phase, and the polarization of the ICRF wave fields are measured and examined. The paper concludes with an overview of the results and summarizes the implications to ICRF coupling in the presence of SOL turbulence. The planned expansion in the diagnostic coverage on ASDEX Upgrade to further address the effects of SOL turbulence on ICRF wave fields is also provided. 


\section{Sector 11 outer wall layout}
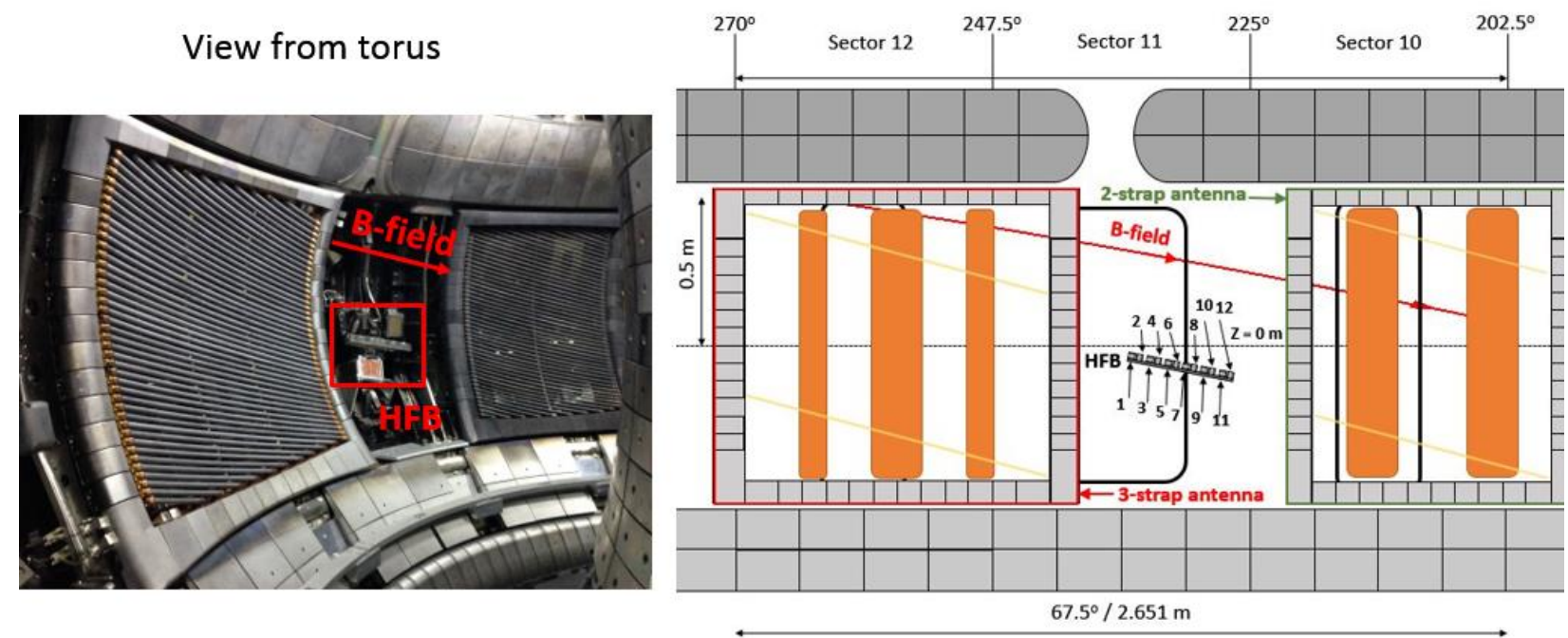

Fig. 1. Layout of the HFB diagnostic inside the ASDEX Upgrade tokamak. The diagnostic is positioned on the low field side in Sector 11 just below the midplane, at the radial position $\sim 10 \mathrm{~cm}$ in the shadow of the ICRF antenna limiter. The numbers " 1 " through " 12 " refer to the individual B-dot probes of the HFB probe array ${ }^{11}$. The tilted lines across the antenna straps indicate the inclination of the antenna Faraday screen.

\section{DIAGNOSTIC AND EXPERIMENTAL METHOD DESCRIPTION}

In order to investigate the evolution of ICRF wave fields (fast and slow) in the SOL region of a tokamak, an array of B-dot probes $^{11}$ was installed on the low field side of ASDEX Upgrade $^{13}$. The in-vessel position of the HFB diagnostic is shown in Fig. 1 and an in-depth description of the diagnostic can be found in Reference ${ }^{11}$. The diagnostic is positioned on the low field side in Sector 11 just below the midplane, at the radial position $\sim 10 \mathrm{~cm}$ in the shadow of the ICRF antenna limiter. The numbers " 1 " through " 12 " refer to the individual B-dot probes of the HFB probe array ${ }^{11}$. The diagnostic is designed to measure the following quantities: 1) the amplitude of the local ICRF wave fields; 2) the parallel (to the background B-field) wave number of the local ICRF wave fields; and 3) the polarization (parallel or perpendicular to the local B-field) of the local ICRF wave fields. The odd numbered probes are oriented to measure the parallel RF $\mathrm{B}$-field component and the even numbered probes are oriented to measure the perpendicular (poloidal) RF B-field component. The RF detector used for RF-to-DC conversion has a built-in bandpass filter ${ }^{11}$, making the diagnostic sensitive only to the 10 $50 \mathrm{MHz}$ frequency range. The diagnostic is positioned toroidally between a 3-strap and a 2-strap ICRF antenna. The 3-strap antenna can be operated at an arbitrary phase and amplitude ratio between the central and the side straps ${ }^{14}$, while the 2-strap antenna operates as a classical dipole antenna ${ }^{15}$. The individual probes in the probe array were absolutely calibrated by measuring known ICRF fields at the radial position of the outer conductor of a matched $50 \mathrm{Ohm}$ coaxial line energized with a $120 \mathrm{~W} \mathrm{RF}$ source ${ }^{11}$. A stainless steel cover with a narrow slit is used to shield the inductor from electrostatic fields, see Fig. 4 in Reference ${ }^{11}$.

The typical operating parameters of the ASDEX Upgrade tokamak $^{13}$ are: the on-axis toroidal $\mathrm{B}$-field $\mathrm{B}_{\mathrm{T}}=-2.5 \mathrm{~T}$, the plasma current $\mathrm{IP}=1 \mathrm{MA}$, the edge safety factor $\mathrm{q} 95=4.4$, deuterium main ion species, the hydrogen minority concentration $\sim 5-10 \%$, the H-mode discharge type, and the diverted lower single null magnetic configuration. The operating frequency of the ICRF antennas in these discharges is $36.5 \mathrm{MHz}$ and the heating scheme is hydrogen minority. The global presence of SOL turbulence in the form of edge localized modes (ELMs) ${ }^{16}$ is measured with grounded current shunts mounted in the divertor region. Measurements of the local ELM-induced plasma density perturbations in the vicinity of the HFB diagnostic are presently not available. The evolution of the edge pedestal region during ELMs is estimated with the integrated data analysis (IDA) routine ${ }^{17}$. The overall results of the diagnostic measurements and data analysis are presented next.

\section{EXPERIMENTAL RESULTS AND DISCUSSION}

ICRF wave field measurements were performed both in vacuum (no plasma, $\mathrm{B}_{\mathrm{T}}=0 \mathrm{~T}$ ) and in an ELMy $\mathrm{H}$-mode plasma. The H-mode discharge was maintained with $5 \mathrm{MW}$ of neutral beam heating and $3 \mathrm{MW}$ of electron cyclotron resonance heating. The ICRF power was varied between 0 and $0.5 \mathrm{MW}$ so as to avoid excessive plasma heating by ICRF waves.

\section{A. Vacuum results}



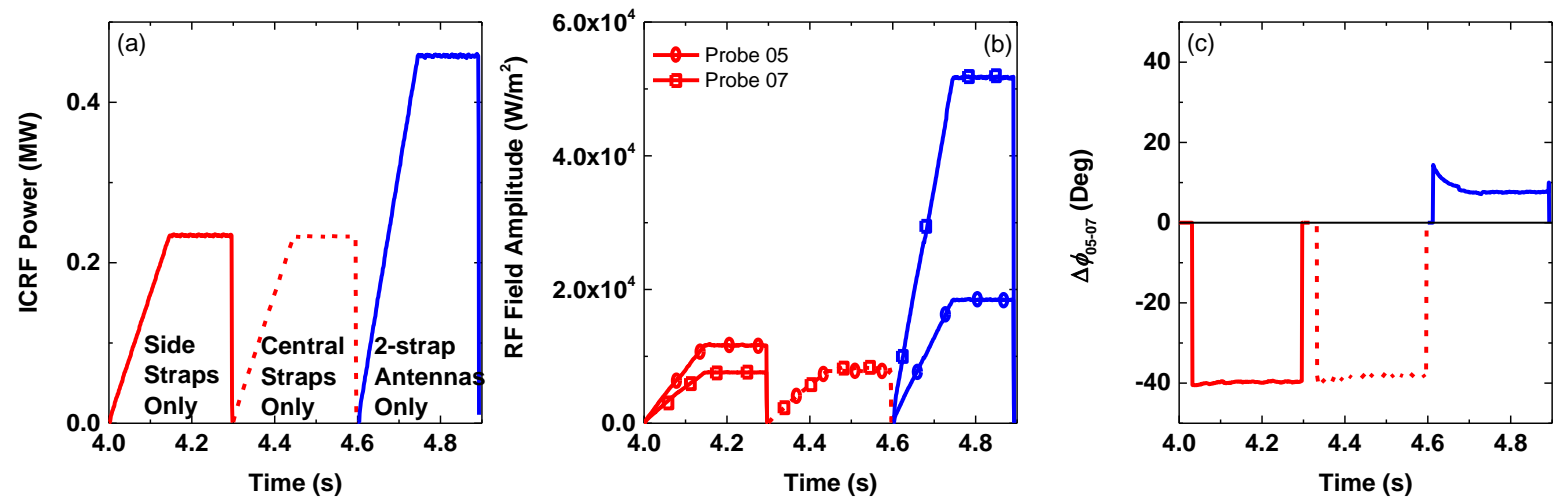

Fig. 2. The response of the HFB probes 05 and 07 as a function of the applied ICRF power under vacuum conditions. The ICRF power traces are shown in (a); probes 05 and 07 amplitude response is shown in (b); and the phase between probes 05 and 07 is shown in (c).

First and foremost, it is important to know what kind of a response the probes have as a function of the applied ICRF power: does it remain linear as in the case of a coaxial line during the calibration procedure ${ }^{11}$ or is it non-linear, perhaps due to additionally induced RF currents in the ASDEX Upgrade vacuum structures? A typical vacuum response of a pair of the HFB probes is shown in Fig. 2. The ICRF power was linearly ramped to $230 \mathrm{~kW}$ for the cases of the side straps only and the central straps only and to $460 \mathrm{~kW}$ for the case of the 2-strap antennas (Fig. 2 (a)). The amplitude response of the individual probes, when expressed in units of power, is linearly proportional to the applied ICRF power (Fig. 2 (b)), while the phase difference between the probes remains independent of the applied ICRF power (Fig. 2 (c)), as long, as the two amplitudes are above their threshold value ${ }^{11}$.

From the principle of operation of the newly installed 3strap antenna, we expect to see outside the antenna box the same spatial distribution of both the amplitude and the phase values when we power the central straps only or the side straps only ${ }^{14}$. Such patterns are, in fact, observed when we power the central straps and the side straps separately, see Fig. 3 (a) and (b). These patterns differ significantly from the RF field distribution inside the active antenna box (Fig. 4), as measured with a portable RF meter before the plasma operations ${ }^{11}$. Note, that the field patterns of the 3-strap antenna cases are distinct from the field patterns generated by a balanced (dipole) 2-strap antenna outside the antenna box (Fig. 3 (a) and (b)). This result is also expected due to the difference in the odd/even symmetry of the two antenna types.

Since the HFB diagnostic is located outside the antenna limiter structure, it is not clear how much the surrounding conducting structures (diagnostics, cables, HFB probes themselves, etc.) influence the excited ICRF field structure in the torus. To assist with the analysis of ICRF wave field distributions in the ASDEX Upgrade torus, a COMSOL-based simulation routine is being developed ${ }^{12}$. The routine is capable of generating a steady-state solution for the ICRF wave field distribution in vacuum (no background magnetic field and no plasma). The boundaries of the numerical simulation are as follows: 1) the vacuum vessel is modeled with an ideal conducting boundary, the shape of which matches the shape of the ASDEX Upgrade plasma facing surface; 2) the key largest torus structures, such as the limiters, the ICRF antenna straps, and the vessel ports, are included (Fig. 3 (c)); 3) no small scale conducting structures, such as other diagnostics, cables, or the probes themselves are included presently. The resulting simulated toroidal vacuum field distribution (Fig. 3 (a)) is distinct from the measured distribution, however, the present COMSOL model does not include smallscale structures in the vicinity of the measured location (other diagnostics, cables, the probes themselves, etc.) and we expect the field patterns to be influenced by the nearest metallic boundaries. The present model is currently being expanded to include small-scale structures in order to match the realistic
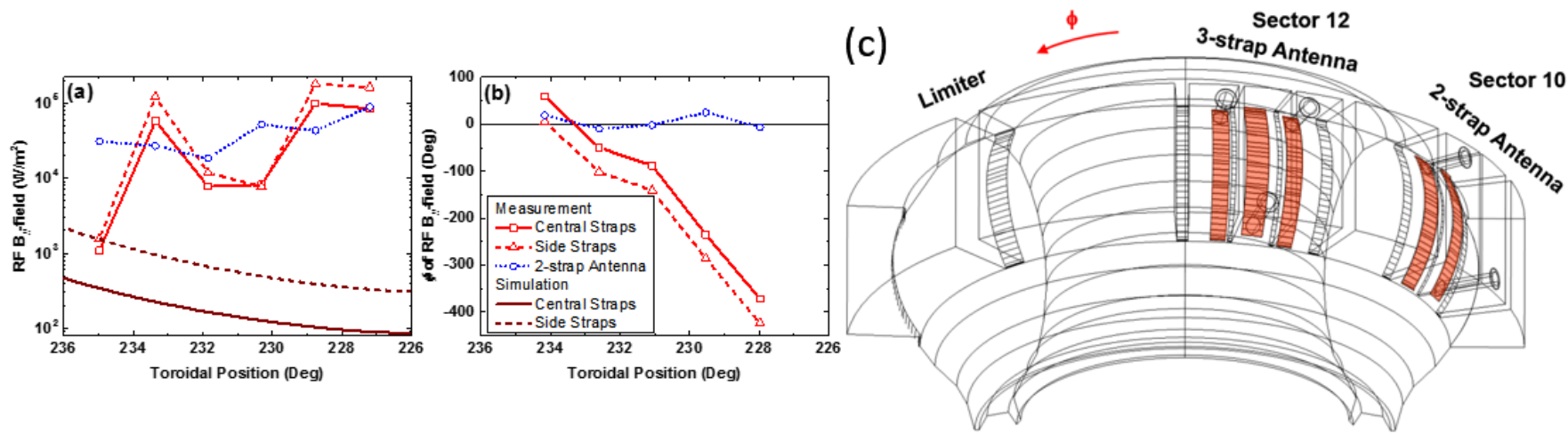

Fig. 3. The toroidal distribution of key parameters measured by the HFB diagnostic. The RF B//-field amplitude distribution, measured and simulated, is shown in (a); the measured phase distribution of the induced ICRF field is shown in (b); and the COMSOL boundary structure used to simulate vacuum ICRF wave fields on ASDEX Upgrade is shown in (c). 
(a)

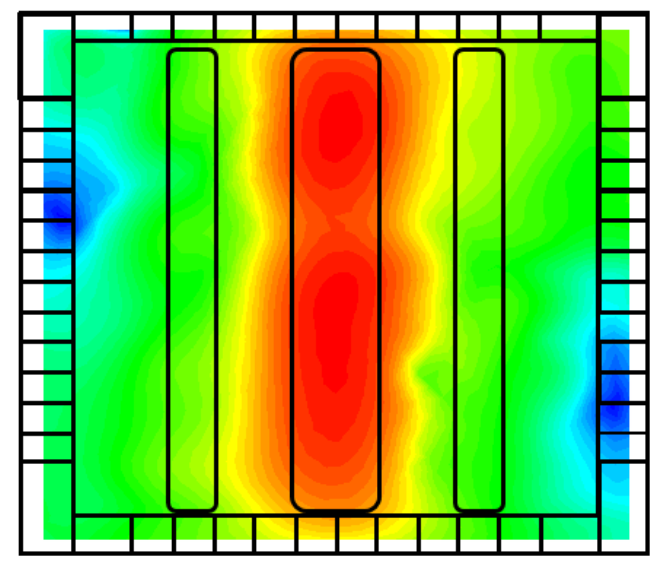

(b)

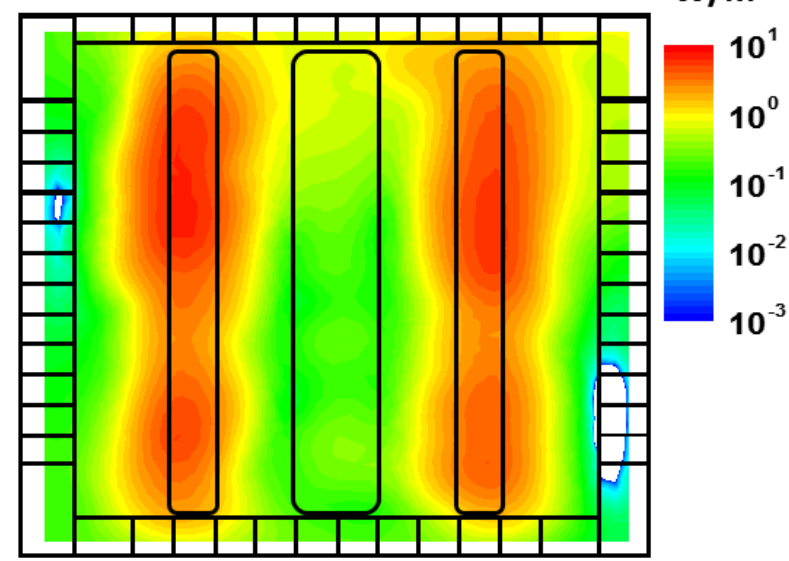

Fig. 4. The measured spatial (in-air) RF power density distribution for the cases of only the central straps being active (a) and only the side strap being active (b). The applied RF power was $10 \mathrm{~W}$ in each case.

boundary conditions in the torus.

\section{B. Plasma results}

The purpose of the HFB diagnostic is to help study ICRF wave fields in the presence of plasma, and a typical signal response during an ELMy H-mode discharge is shown in Fig. 5. The applied ICRF power waveform (Fig. 5 (a)) is the same as for the vacuum case (Fig. 2 (a)). Note that, at first, the amplitude and the phase measurements (Fig. 5 (b) and (c)) appear to be "noisy" compared to their vacuum values (Fig. 2 (b) and (c)). The goal of this section of the paper is to demonstrate that we are dealing with a turbulent perturbation of the ICRF fields caused by the appearance of plasma filaments in the SOL region. Also note, that the launched ICRF power is modulated during the discharge (Fig. 5 (a)); however, these modulations are on the order of $\sim 10 \%$ of the total power. Since the probe amplitude response is linear with power (Fig. 2 (a) and (b)), these modulations are not sufficient to account for the observed changes of $>100 \%$ (Fig. 5 (b)). Something else must be playing a role during plasma discharges.

One of the defining features of steady $\mathrm{H}$-mode plasmas is the presence of ELMs ${ }^{13}$. A detailed probe amplitude and phase response during several ELM cycles is shown in Fig. 6 (a), (b), and (c). The presence of ELMs is registered by measuring the

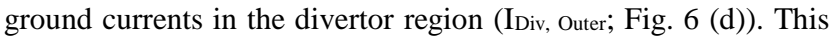
measurement allows us to clearly define the "During-ELM" and the "Inter-ELM" periods: the sharp spikes in the current values are the plasma density perturbations that happen during ELM events. The ICRF wave field measurements can now be separated into two categories: during the "Inter-ELM" period the measured wave fields have a well-defined phase distribution (Fig. 7), consistent with the wave field distribution at the probe location dominated by a single source with a well-defined phase. However, during the ELMs the phase measurement becomes "scattered" (Fig. 7): the wave field distribution at the probe location is now consistent with a sum from multiple sources, each with a random phase to each other. A close examination of the plasma density profile evolution localizes these sources to be the plasma density perturbations introduced by the ELMs into the SOL region (Fig. 6 (e)). The plasma pedestal region (located at the normalized poloidal radius coordinate $\rho_{\mathrm{P}}=0.96$, $\rho_{\mathrm{p}} \equiv\left[\left(\Psi-\Psi_{\text {axis }}\right) /\left(\Psi_{\text {separatrix }}-\Psi_{\text {axis }}\right)\right]^{1 / 2}$, where $\Psi$ is the poloidal flux $)$ relaxes during ELM events, i.e. the plasma density suddenly drops in value. This density drop is accompanied by a plasma density rise in the SOL region. The density rise is not spatially uniform but is in the form of poloidally localized, field-aligned filaments ${ }^{13}$. Recent EMC3-EIRENE simulations confirmed that poloidal plasma density perturbations in the SOL can influence ICRF wave coupling ${ }^{18}$. Note that the plasma density values in these filaments are often high enough for the fast ICRF wave to become propagating within the filaments (Fig. 6 (e)). The boundary between propagation and evanescence is defined by the condition when the perpendicular index of refraction $n_{\perp}$ in the cold plasma dispersion relation is equal to zero. This relation

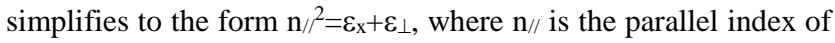
refraction of the launched ICRF fast wave and $\varepsilon_{\mathrm{x}}$ and $\varepsilon_{\perp}$ are the off- and on-diagonal components of the dielectric tensor ${ }^{19}$. The
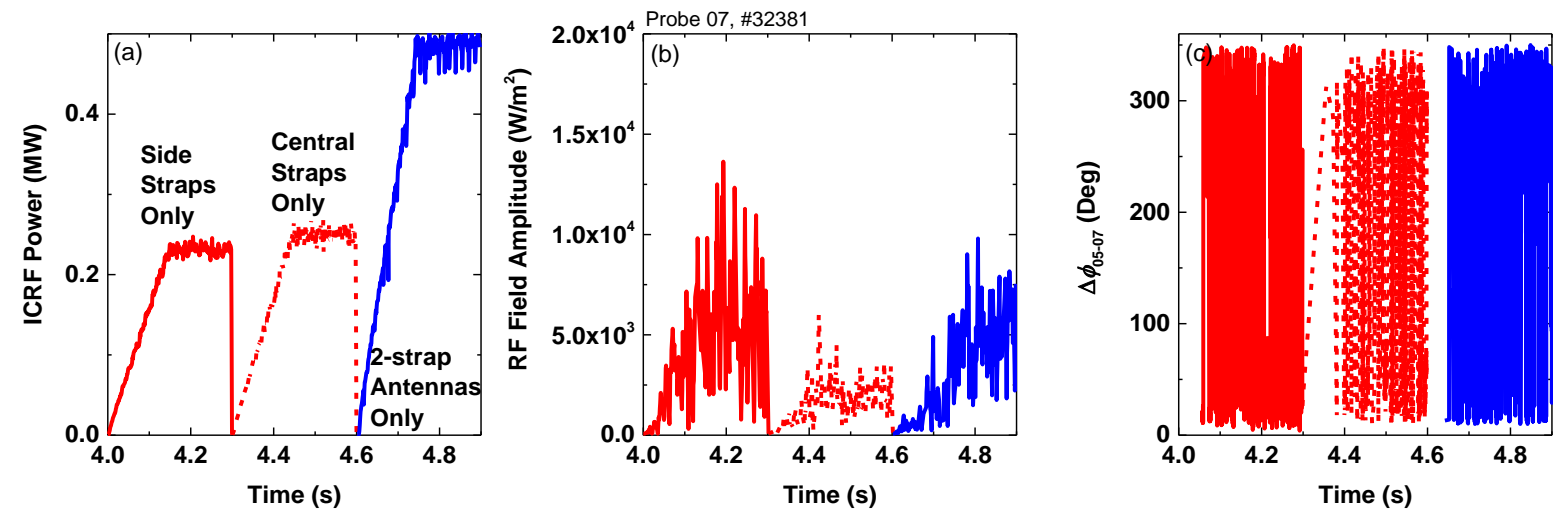

Fig. 5. A typical response of the HFB probe signals in ELMy H-mode plasmas. The applied ICRF power is shown in (a); the measured HFB probe 07 amplitude response is shown in (b); and the measured HFB phase response between probes 05 and 07 is shown in (c). 

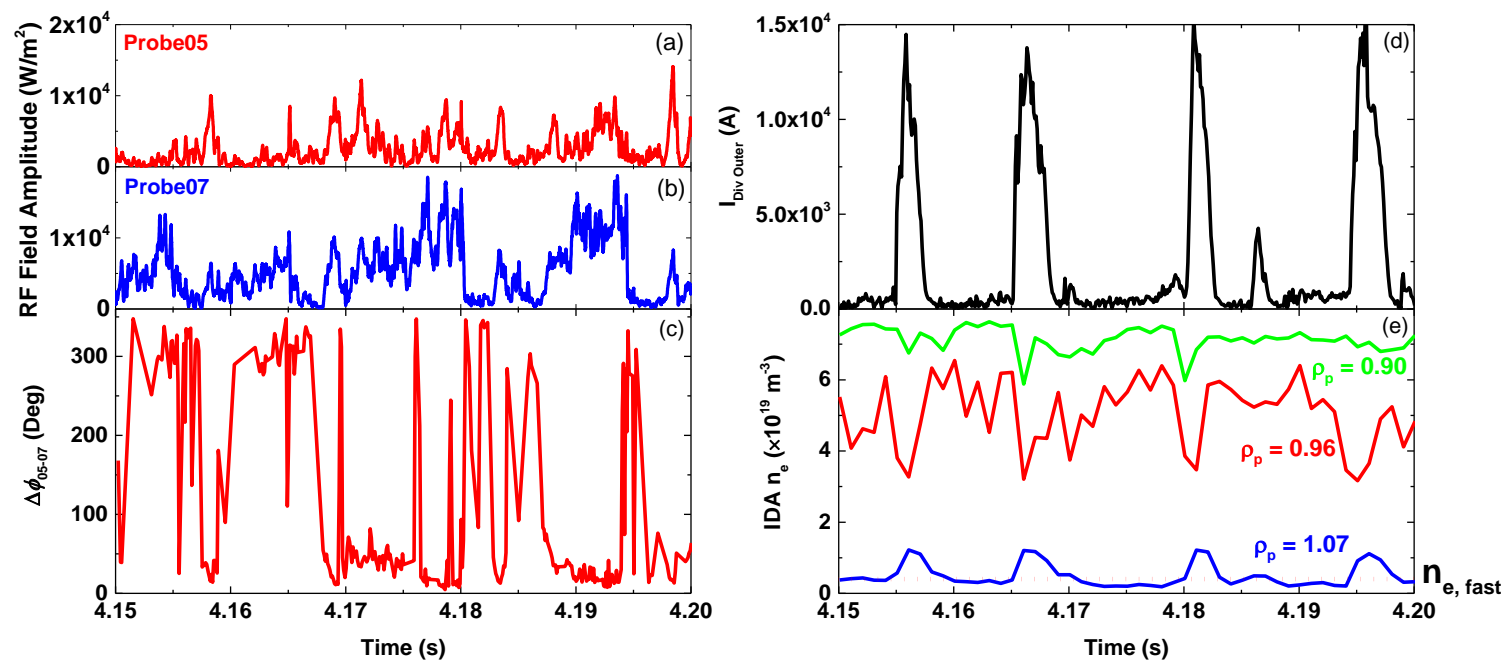

Fig. 6. A detailed view of the amplitude and the phase response of the HFB probe signals during a typical ELMy H-mode discharge. The

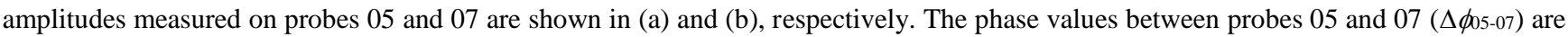
shown in (c); the response of the outer divertor current shunts (I $\mathrm{I}_{\text {iv Outer }}$ ) is shown in (d); and the IDA-estimated plasma density ( $\mathrm{n}_{\mathrm{e}}$ ) evolution in the pedestal region $\left(\rho_{p}=0.96\right)$, just inside the pedestal region $\left(\rho_{p}=0.90\right)$ and just outside the pedestal region $\left(\rho_{p}=1.07\right)$ are shown in (e). The red dotted line in (e) shows the expected plasma density value of the fast wave cut off ( $\mathrm{n}_{\mathrm{e}}$, fast) from the cold plasma dispersion relation.

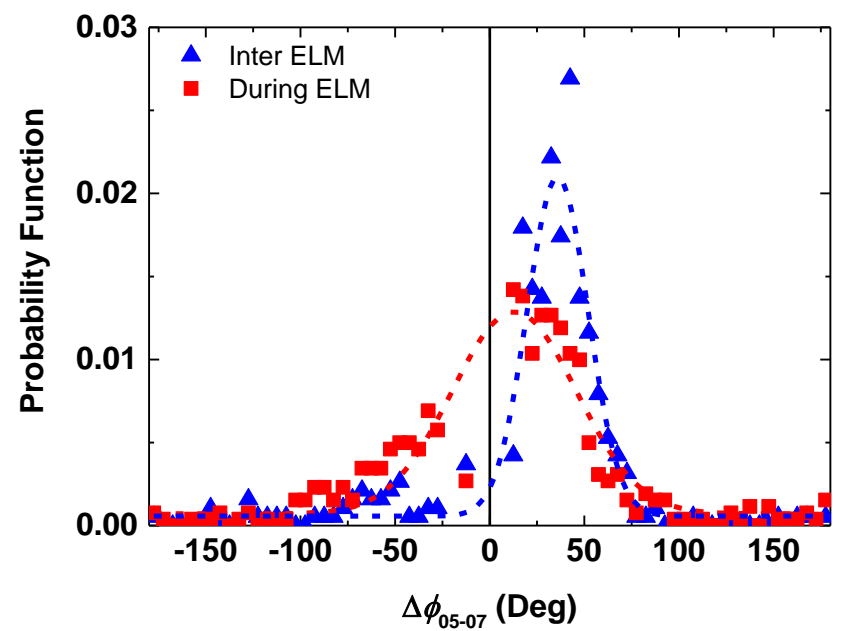

Fig. 7. The probability distribution of the phase measurement between probes 05 and 07 in an ELMy H-mode discharge. The green solid triangles are for the "Inter-ELM" period and the red solid squares are for the "During-ELM" period. The dashed lines are Gaussian fits to guide the eye only.

value of the fast wave cut off density $n_{e}$, fast is then calculated and shown in Fig. 6 (e) for the particular case of ASDEX Upgrade antennas $\mathrm{n}_{/ /=13}$. Also note, that the plasma density perturbations during ELMs are greatly diminished inside the pedestal region ( $\rho_{\mathrm{P}}<0.90$, Fig. 6 (e)), suggesting that it is the SOL physics, not the core, that plays a key role in modulating the observed ICRF wave field signals (Fig. 5).

\section{CONCLUSION AND FUTURE WORK}

A dedicated diagnostic was installed on ASDEX Upgrade with the goal of studying ICRF wave fields in the SOL during plasmas (Fig. 1). The diagnostic consists of 6 B-dot probe pairs capable of measuring the amplitude, the phase, and the polarization properties of ICRF wave fields. The diagnostic is sensitive to the $10-50 \mathrm{MHz}$ frequency range. A series of vacuum measurements $\left(\mathrm{B}_{\mathrm{T}}=0 \mathrm{~T}\right.$, no plasma) outside the antenna box reveal that the spatial RF field pattern imposed by the central straps of the 3-strap antennas is nearly identical to the spatial pattern imposed by the side straps of the 3 -strap antennas (Figs. 2 and 3). This result is expected from the operating principle of the 3-strap antenna. However, the non-monotonic amplitude variations observed between individual probes (Fig. 3 (a)) are not expected from a simple vacuum picture. In order to further improve our understanding of the excited vacuum fields, a COMSOL-based numerical model of the ASDEX Upgrade torus is being developed with early results showing the importance of nearby conducting structures on the wave field measurements (Fig. 3). The excited vacuum fields become strongly modified during ELMy H-mode plasmas, these modifications are not due to the variations in the coupled ICRF power from the active antennas (Figs. 5 and 6). In fact, these modifications are consistent with SOL density perturbations caused by poloidally localized filaments, density perturbations strong enough to affect fast wave coupling (Fig. 6). The ICRF measurements during ELMs show a field structure consistent waves scattered from multiple sources, each with a random phase correlation to each other (Fig. 7).

In order to further explore the interactions between ICRF wave fields and SOL plasmas, the HFB diagnostic is being expanded. The new features include: 1) additional probe pairs on the low field side and the high field side to reconstruct the global ICRF wave field distribution in the torus; and 2) a probe pair on the low field side manipulator to reconstruct the wave field radial profile across the SOL region. The results from these new additional probes will be presented at a future conference.

\section{ACKNOWLEDGEMENTS}

This work has been carried out within the framework of the EUROfusion Consortium and has received funding from the Euratom research and training programme 2014-2018 under grant 
agreement No 633053. The views and opinions expressed herein do not necessarily reflect those of the European Commission. The author and the co-authors would also like to acknowledge the contributions of Bertram Brucker, Johann Kneidl, and Gerhard Siegl in the design, the manufacture, the assembly, and the installation of the HFB probe array on ASDEX Upgrade.

\section{REFERENCES}

${ }^{1}$ T.H. Stix. Waves in Plasmas. Springer, 1992.

${ }^{2}$ W. Horton. Turbulent Transport in Magnetized Plasmas, 2012.

${ }^{3}$ S. J. Zweben, J. A. Boedo, O. Grulke, C. Hidalgo, B. LaBombard, R. J.

Maqueda, P. Scarin and J. L. Terry, Plasma Phys. Control. Fusion 49, 7 (2007).

${ }^{4}$ E. Ott, Phys. Fluids 22, 9 (1979).

${ }^{5}$ P.T. Bonoli and E. Ott, Phys. Rev. Lett. 46, 6 (1981).

${ }^{6} \mathrm{M}$. Ono, Phys. Fluids 26, 6 (1982).

${ }^{7}$ J. D. Moody, M. Porkolab, C. L. Fiore, F. S. McDermott, Y. Takase, J.

Terry, and S. M. Wolfe, Phys. Rev. Lett. 60, 4 (1988).

${ }^{8}$ V. Pericoli-Ridolfini, L. Giannone, R. Bartiromo, Nuclear Fusion 34, 4 (1994).

${ }^{9}$ J. R. Myra and D. A. D’Ippolito, Phys. Plasmas 17, 102510 (2010).

${ }^{10}$ J. R. Myra, Phys. Plasmas 21, 022507 (2014).

${ }^{11}$ R. Ochoukov, V. Bobkov, H. Faugel,1 H. Fünfgelder, J.-M.

Noterdaeme, and ASDEX Upgrade Team, Rev. Sci. Instrum. 86, 115112

(2015).

${ }^{12}$ https://www.comsol.com/

${ }^{13}$ H. Zohm et al., Nucl. Fusion 55, 104010 (2015).

${ }^{14} \mathrm{~V}$. Bobkov et al., submitted to Nucl. Fusion.

${ }^{15}$ V. Bobkov et al., Nuclear Fusion 53, 093018 (2013).

${ }^{16} \mathrm{H}$. Zohm, Plasma Phys. Control. Fusion 38, 105-128 (1996).

${ }^{17} \mathrm{R}$ Fischer, C J Fuchs, B Kurzan, W Suttrop, and E Wolfrum, Fusion

Science and Technology 58 (2):675-684 (2010).

${ }^{18}$ W. Zhang et al, Nucl. Fusion 56, 035007 (2016).

${ }^{19}$ D.A. D'Ippolito, J.R. Myra, E.F. Jaeger, and L.A. Berry, Phys. Plasmas 15, 102501 (2008). 